Sollen gedoppelte Stoffe nach diesem Verfahren vulkanisirt werden, so ist obige Maschine nicht verwendbar, da bei dieser Art wasserdichter Stoffe der Kautschuküberzug beiderseitig von Stoff bedeckt ist. Solche Stoffe lassen sich nach diesem Verfahren nux rulkanisiren, wenn die Vulkanisirung gleichzeitig mit der Operation des Doppelns geschieht.

$\mathrm{Zu}$ diesem $\mathrm{Z}_{\text {weck }}$ dient die in Fig. 272 in schematischer Darstellung skizzirte $\mathrm{Ma}$ schine. Dieselbe besteht aus zwei schweren Papierwalzen $a$ und $b$, nach Art der Kalanderwalzen. Die Walze $b$ ist parallel zur Walze $a$ beweglich und wird mittels auf Hebel wirkender Gewichte kräftig gegen dieselbe angepresst. $d$ und $c$ sind die Walzen, auf denen die zu doppelnden Stoffe aufgebäumt sind. Die gedoppelten Stoffe werden auf der Walze $g$ aufgewickelt. $e$ ist

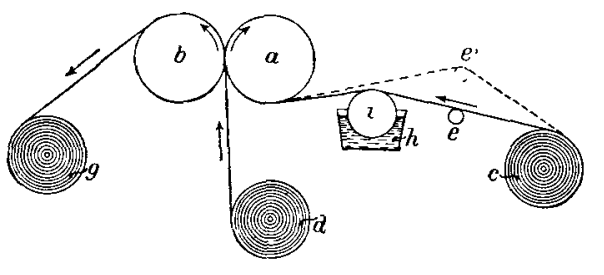

Fig. 272.

eine Leitwalze und kann mittels eines Hebels gehoben $\left(e^{1}\right)$ oder gesenkt werden, so dass der von $d$ sich abwickelnde Stoff nach Belieben über oder auf der in dem Trog $h$ liegenden Walze $i$ hinweggeführt werden kann. Sollen nun Stoffe gedoppelt werden, die für heisse Vulkanisation bestimmt sind, also den erforderlichen Schwefel bereits in der Kautschukmischung enthalten, so werden einfach die Stoffe, wie sie von der Auftragmaschine kommen, an die Stelle der Walzen $c$ und $d$ eingesetzt, so dass sie sich mit ihren Kautschukseiten zwischen den Kalanderwalzen $a$ und $b$ treffen. In Folge des durch die Walzen ausgeübten starken Druckes vereinigen sich die beiden getrennten Kautschukflächen zu einer einzigen homogenen Schicht. Während dieser Operation wird die Leitwalze $e$ nach $e^{1}$ verstellt. Sollen die Stoffe aber mit Schwefelchlorür vulkanisirt werden, so werden dieselben wie vorher in die Maschine eingesetzt, der von der Walze $d$ kommende Stoff wird aber nunmehr über die in der Lösung von Schwefelchlorür in Schwefelkohlenstoff im Troge $h$ rotirende Walze $i$ geführt, dadurch mit der Lösung benetzt und noch ehe der aufgenommene Schwefelkohlenstoff Zeit hatte, zu verdampfen, findet die Vereinigung der beiden Stoffe zwischen den Kalanderwalzen statt. Das Vulkanisirungsmittel diffundirt gleichmässig in die beiden Kautschukschichten und bewirkt sodann deren Vulkanisation. Die Concentration der Schwefelchlorürlösung muss in diesem Falle natürlich stärker genommen werden, da die auf einer der sich vereinigenden Kautschukflächen aufgetragene Menge des Vulkanisirungsmittels für die Vulkanisirung der beiden sich vereinigenden Flächen ausreichen muss. Es wird daher eine Concentration von 8:200 in diesem Falle angewandt. Die auf diese Art vulkanisirten gedoppelten Stücke werden ebenfalls noch durch die Heissluftkammer zur Geruchlosmachung genommen.

Im Vorstehenden sind alle Punkte, die bei der Fabrikation wasserdichter Gummistoffe von Wichtigkeit sind, in Betracht gezogen worden. Die nach diesen Methoden hergestellten Kautschukflächen, soweit dieselben sichtbar sind, besitzen stets eine mehr oder weniger indifferente Farbe, die gewöhnlich zwischen braun und gran liegt, je nach dem dem Kautschuksatze zugesetzten Pigmente. Soweit die gedoppelten Stoffe in Betracht kommen, ist die Farbe des dazwischen liegenden Kautschuks natürlich unerheblich. Gedoppelte Stoffe besitzen aber, besonders wenn sie aus dünnern Stoffen hergestellt wurden, eine unangenehme Steifheit, in Folge dessen daraus hergestellte Mäntel den berüchtigten steifen Faltenwurf besitzen. Für Damenmäntel bestimmte Stoffe werden deshalb jetzt überhaupt nicht mehr gedoppelt, sondern lediglich mit einseitiger Kautschukfläche versehen. In diesem Falle liegt der Gedanke sehr nahe, die unschöne Farbe der Kautschukseite durch dem Wechsel der Mode folgende Ornamentirungen zu beleben. Die dazu benutzten Verfahren sind natürlich der Beschaffenheit der zu ornamentirenden Fläche und ihrer physikalischen und chemischen Natur entsprechend, ganz eigenthümlicher Art und gehören ausnahmslos alle der Neuzeit an. Die Beschreibung dieser Verfahren, die meistens sehr gewinnbringend für die Erfinder waren oder noch sind, soll binnen Kurzem folgen.

\section{Über Ekenberg's Raffinationsverfahren von ölen, Fetten u. dgl. \\ Von}

\section{Rudolf Benedikt.}

Martin Ekenberg hat im Anschlusse an seine Versuche mit dem Centrifugalemulsor (d. Z. 1892, 486) der Actiebolaget Separator in Stockholm ein Raffinationsverfahren für 
Öle ausgearbeitet. Ich habe Gelegenheit gehabt, dasselbe in Stockholm im Laboratorium der genannten Gesellschaft kennen zu lernen und in Wien mit mir von derselben zur Verfügung gestellten Apparaten eingehend zu studiren. Ausserdem verdanke ich Herrn Ekenberg eine Reihe von Mittheilungen darüber. Bei der Bedeutung, welche dieses Verfahren in nicht allzulanger Zeit gewinnen dürfte, sei es mir gestattet, dasselbe hier in Kurzem zu beschreiben.

Der Centrifugalemulsor gestattet bekanntlich, Flüssigkeiten auf das Innigste mit einander zu emulgiren, so dass ex mit Vortheil einerseits zum Vermischen von fetten Ölen, geschmolzenen Fetten, Theerölen u. s. w. mit Schwefelsäure, mit Alkalilösungen u. dgl., andererseits zum Waschen dieser Öle mit Wasser benutzt werden kann.

Die erhaltenen Emulsionen werden, insofern sich dieselben nicht beim blossen Stehen wieder vollständig entmischen, mittels des de Laval'schen Separators getrennt.

Ekenberg's System zur Reinigung von Ölen besteht nun darin, dass dieselben continuirlich durch ein System von Emulsoren und Separatoren hindurchlaufen und auf diesem Wege der Einwirkung der Reagentien sowie den erforderlichen Waschungen unterworfen werden.

Die Combination von je einem Emulsor und einem Separator bildet ein Waschelement. In solchen Fällen, wo die Emulsion sich so leicht scheidet, dass kein Centrifugalseparator erforderlich ist, tritt an dessen Stelle eine Florentinervorlage. Die Anzahl von Waschelementen, welche zu einer Waschbatterie zusammengestellt werden, ist von der Anzahl der Waschungen abhängig.

In der Regel fliesst die Emulsion aus dem Emulsor direct in den Separator, da die Reaction zwischen den flüssigen oder in Lösung befindlichen Reinigungsmitteln und den zu entfernenden Bestandtheilen der Öle sich in Folge der ausserordentlich innigen Berührung sofort vollzieht. Sollte in einzelnen Fällen eine längere Einwirkung erwünscht sein, so führt man einfach zwei Reservoire in die Reihe ein, welche die Mischung abwechselnd aufnehmen. Wollte man die Mischung z. B. 24 Stunden stehen lassen, so müsste man die Gefässe so gross wählen, dass jedes im Stande wäre, die Tagesproduction aufzunehmen. Während man die Emulsion in das eine Gefäss laufen lässt, verarbeitet man im Separator die im andern Gefässe befindliche, am Vortage hergestellte Mischung. Die Regulirung des Mischungsverhältnisses geschieht entweder in der Weise, dass man die Ausflussöffnungen des zwei- kammerigen Zuflussgefässes mittels eingesetzter konischer Regulirstifte passend verengt, oder bei grösseren Anlagen mittels Pumpen von bekannter Leistung.

Zur schnellen Erhitzung oder Kühlung werden Plattenvorwärmer oder -kühler eingeschaltet.

Zur Transportirung der Flüssigkeiten von einem Waschelement zum andern werden Centrifugalpumpen benutzt, welche direct von der Emulsor- oder Separatorwelle getrieben werden und demnach immer gleichzeitig mit diesen Maschinen arbeiten.

Eine etwa erwünschte Oxydation mit Luft oder Desodorisation mit trockenem Dampf will Ekenberg mit einem neu erfundenen Gasemulsor ausführen, über dessen Construction demnäcbst Näheres mitgetheilt werden soll.

Die Leistungsfähigkeit einer Waschbatterie mit Emulsoren und Separatoren gewöhnlicher Grösse beträgt je nach der Natur des Öles, der Art der Reinigung, den Mischungsverhältnissen und der Combination der Apparate täglich 3 bis $6000 \mathrm{k}$. Nur wenn sehr stark säurehaltige fette Öle bis zu 25 Proc. freie Fettsäuren - gereinigt werden sollen und demgemäss grössere Mengen der verdünnten Laugenlösung zum Waschen nothwendig sind, sinkt die Leistungsfähigkeit noch mehr herab.

Die Teller der Emulsoren und die Separatortrommeln sind aus Säurestahl hergestellt, welcher ausserordentlich widerstandsfähig gegen concentrirte Schwefelsäure und gegen Laugen ist. Für verdünnte Säuren werden Bronzeteller und durch Eintauchen in die geschmolzene Legirung mit Bleiantimon überzogene Separatortrommeln benutzt.

Zur Erläuterung des Verfahrens seien einige Beispiele angeführt.

\section{Raffiniren von Vaselinölen und schweren Mineralölen.}

Zum Raffiniren dieser Öle werden wie gewöhnlich 8 bis 25 Proc. Schwefelsäure angewendet und das Öl hierauf mit verdünnter Lauge und endlich mit Wasser gewaschen.

In dem Emulsor $S_{1}$ (Fig. 273) $^{1}$ ) wird das aus dem Behälter 1 kommende Rohöl mit vom Separator abfliessender, schon einmal benutzter Säure innig gemischt. Die Mischung gelangt in den Separator $E_{1}$, welcher die nun zweimal benutzte Säure abscheidet und in den Behälter 2 leitet. Die Quantität der Schwefelsäure hängt von der Qualität des Öles ab. Diese Behandlung

1) Die Bezeichnungen sind in der Zeichnung für die erste Waschbatterie ( $E$ für Emulsor, $\boldsymbol{S}$ für Separator) vertauscht. 
mit der benutzten Schwefelsäure, welche durch die erste Operation von 1,84 auf 1,5 bis 1,6 spec. G. verdünnt wurde, bewirkt vornehmlich eine Entwässerung des Öles.

Das entwässerte Öl wird im Emulsor $E_{2}$ mit frischer Säure aus dem Behälter 3 gemischt, die Mischung im Separator $S_{2}$ abseparirt und die gebrauchte Säure zum Emulsor $S_{1}$ geleitet.

In den Waschelementen $E_{3} S_{3}$ und $E_{4} S_{4}$ wird das Öl mit Lauge gewaschen. Im Reservoir 6 befindet sich frische, in 5 zweimal benutzte Lauge. In Fällen, in welchen ein zweites Waschen mit Lauge übertlüssig erscheint, schaltet man das Element $E_{4} S_{4}$ aus. Endlich wäscht man im Emulsor $E_{5}$ mit tiger Einstellung kommt selten mehr als 0,1 Proc. Öl in das Waschwasser.

Die zweimal gebrauchte Säure kann mit Wasser verdünnt, vom aufschwimmenden Theer getrennt und z. B. zur Fabrikation von Ammoniumsulfat verwendet werden, die zweimal gebrauchte Lauge lässt sich mit Kalk theilweise regeneriren.

\section{Raffiniren von fetten Ölen.}

Eine derartige Anlage besteht, falls man in gewöhnlicher Weise nur mit Schwefelsäure raffinirt, aus nur drei Waschelementen. Im ersten wird die Schwefelsäure eingemischt und abseparirt, im zweiten wird mit kaltem, im dritten mit warmem Wasser gewaschen.

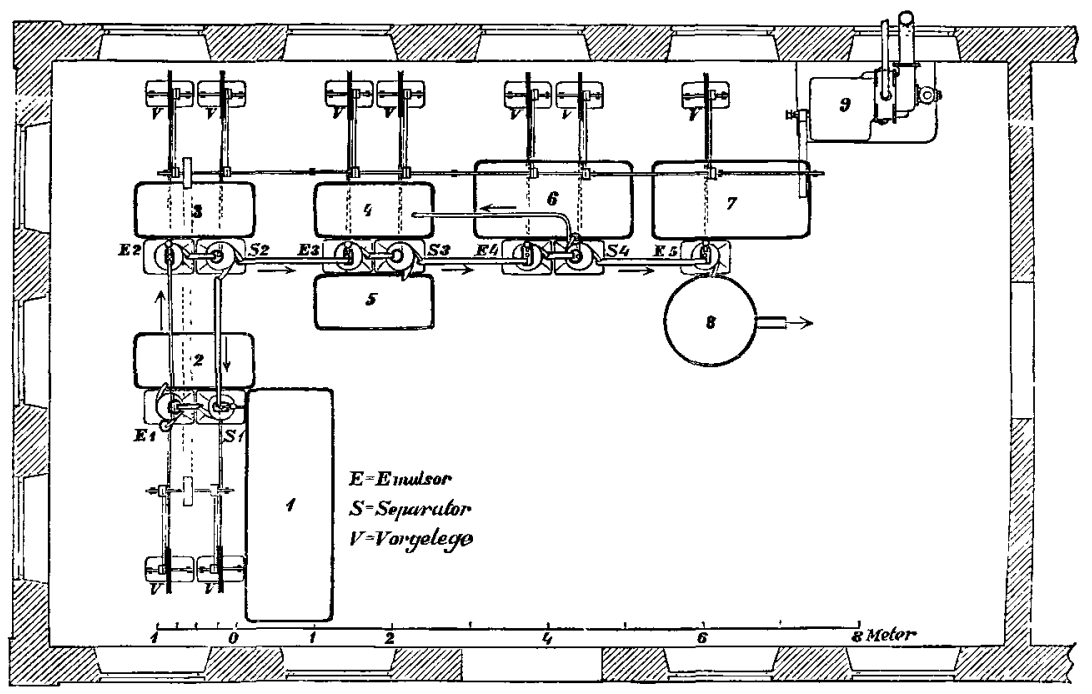

Fig. 273.

warmem aus Reservoir 7 kommenden Wasser und lässt es in der Florentinervorlage $8 \mathrm{ab}-$ sitzen.

Die Dampfmaschine 9 treibt alle Emulsoren und Separatoren.

Bei dieser Art der Behandlung scheidet sich das Säureharz nie in grossen festen Klumpen aus, wie dies sonst häufig vorkommt, sondern es entsteht eine homogene, dickflüssige Harzlösung, welche nur nach längerem Stehen zu einer harten Masse, dem Säureharz, erstarrt.

Die Säureseparatoren sind so eingerichtet, dass sie sich von selbst entleeren, wenn sie in Ruhe versetzt werden. Sie sind einfache Trommelseparatoren, während die Laugenseparatoren $S_{3}$ und $S_{4} \alpha$-Separatoren sind, d. h. Tellereinsätze haben, durch welche die Wirkung bedeutend erhöht wird.

Die Separatoren scheiden namentlich alle dünnflüssigen Mineralölemulsionen sehr scharf und zwar gleich gut, ob die Waschwässer sauer oder alkalisch sind. Bei rich-
$R \ddot{u ̈ b o ̈ l}$ wird nach dem Auspressen erst durch Abstehen von Wasser und Samentheilen geklärt, sonst ist der Schwefelsäureverbrauch ein grösserer. Das Öl läuft nach der letzten Waschung zwar klar ab, trübt sich aber nach wenigen Stunden durch Ausfallen von etwa 0,1 Proc. Wasser, welches man durch neuerliche Separation trennen kann.

Liegt ein an freien Fettsäuren reiches Öl vor, so wäscht man nach Abseparirung der Säure statt mit Wasser mit verdünnter Lauge.

III. Entsäuern stark saurer Fette und fetter Öle.

Man kann mittels des Systems Ekenberg auch vollkommen neutrale Fette herstellen und zwar selbst aus Producten, welche bis zu 25 Proc. freie Fettsäuren enthalten. Die gereinigten Fette sind in vielen Fällen direct als Speiseöle verwendbar, in anderen müssen noch andere nicht saure, übelschmeckende Bestandtheile entfernt werden.

Eine Anlage für die Entsäuerung von Cocos- oder Olivenöl ist in Fig. 274 veran- 
schaulicht. Dieselbe besteht aus sechs Waschelementen, wovon drei an Stelle der Centrifugalseparatoren Florentinervorlagen enthalten.

Das Öl fliesst aus dem Reservoir 1 nach dem Waschelement $E_{1} S_{1}$ und wird dort mit aus dem Behälter 2 kommender Lauge gewaschen.

In $E_{3} S_{2}$ wird es mit Wasser, in $E_{3} S_{3}$ zum zweiten Mal mit Lauge, in $E_{4}$ mit Wasser, in $E_{5}$ mit schwacher Lauge, in $E_{5}$ mit ganz schwacher Säure (Schwefelsäure
Die Separatortrommel hat bekanntlich die Einrichtung einer um eine verticale Achse rotirenden Florentinerflasche. Wird nun auch die Wirkung des Separators in ganz anderer, sehr complicirter Weise berechnet, so lässt sich doch an dem Beispiel der Florentiner andeuten, inwiefern die Einstellung des Separators von Bedeutung ist. Lässt man in eine Florentinerflasche continuirlich gleichzeitig zwei Flüssigkeiten von verschiedenem specifi-

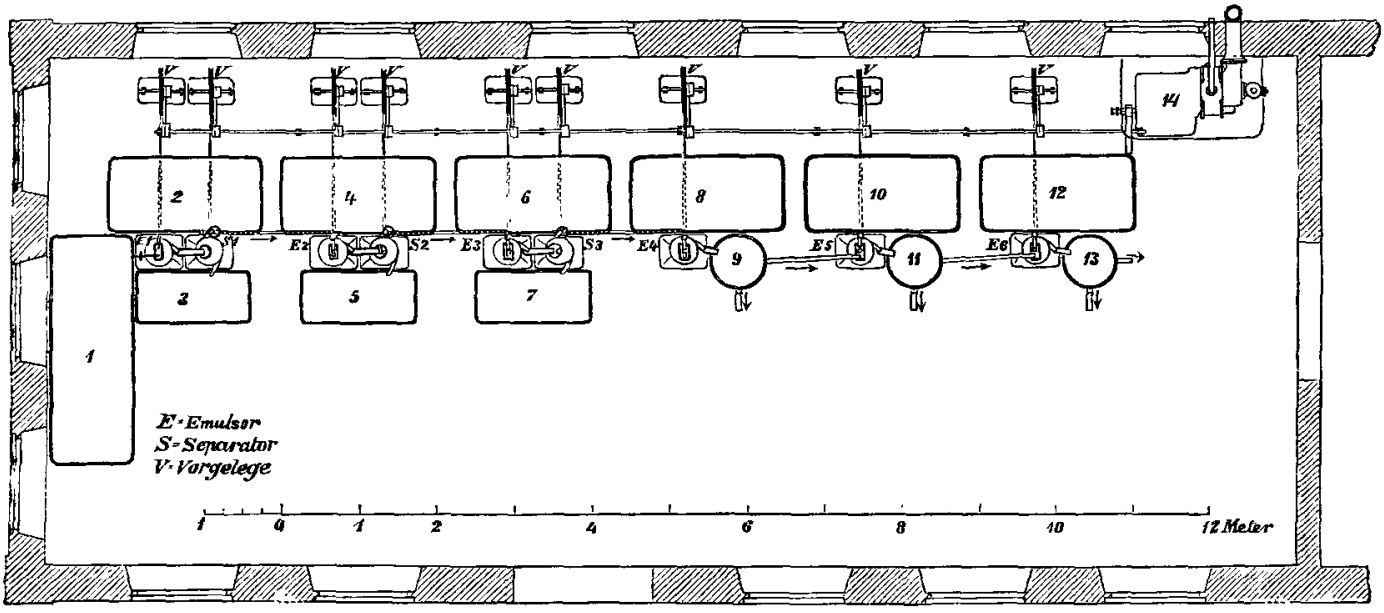

Fig. 274.

oder Salzsäure), endlich in $E_{6}$ mit warmem Wasser gewaschen.

Die Concentration der Lauge ist von den auszuwaschenden Säuremengen und dem Volumverhältniss zwischen Öl und Lauge abhängig und bewegt sich meistens zwischen 0,25 bis 0,5 Proc. Die Reservoire 2 und 6 enthalten Lauge, 4, 8 und 12 Wasser, 10 Säure, während 3,5 und 7 die Seifenlösung auffangen.

Cocosöl muss selbstrerständlich in geschmolzenem Zustande mit vorgewärmten Laugen $u$. dgl. behandelt werden.

Von den grossen Vortheilen, welche das Ekenberg'sche System den alten Raffinationsverfahren gegenüber bietet, wird noch die Rede sein. Es muss aber auch darauf hingewiesen werden, dass man mitunter auf nicht unbedeutende Schwierigkeiten stösst.

Der Centrifugalemulsor arbeitet in allen Fällen, auch wenn der Tellerabstand sehr gross gewählt wird, vorzüglich. Nicht dasselbe kann vom Separator gesagt werden, welcher die Emulsion zuweilen nicht genügend vollständig in ihre Bestandtheile scheidet. Der Grund hiefür kann zunächst in der unrichtigen Einstellung des Separators liegen, indem jeder Separator speciell für die beiden zu trennenden Flüssigkeiten auf Grund der Differenz der specifischen Gewichte eingestellt sein muss. schen Gewicht einfliessen, welche sich rasch von einander scheiden, so fliesst die schwerere durch das Abflussrohr, die leichtere über den Rand des Gefässes $a b$. Sei h die Höhe der Flasche vom Rande bis zum untern Rohransatz,

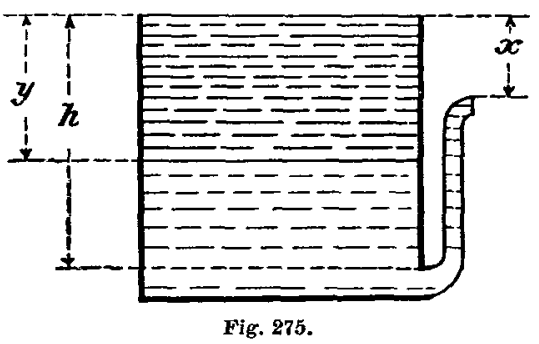

$x$ der Abstand der Ausflussöffnung vom oberen Rande der Flasche, $\mathrm{y}$ die Höhe der leichteren Flüssigkeitsschicht, s deren specifisches Gewicht, s' das specifische Gewicht der schwereren Flüssigkeit, so ist bekanntlich

$$
\begin{aligned}
& \mathrm{sy}+(\mathrm{h}-\mathrm{y}) \mathrm{s}^{\prime}=(\mathrm{h}-\mathrm{x}) \mathrm{s}^{\prime} \\
& \mathrm{y}=\frac{\mathrm{x} \mathrm{s}^{\prime}}{\mathrm{s}^{\prime}-\mathrm{s}} .
\end{aligned}
$$

$\mathrm{y}$ ist also bei gegebenem specifischen Gewicht abhängig von $x$ und es kommt nun zunächst darauf an, den Abstand $x$ so einzujustiren, dass der Apparat möglichst vollständig scheidet. Für $y=h$ erreicht $x$ seinen grössten Werth, nämlich

$$
x_{\max }=\frac{h\left(s^{\prime}-s\right)}{s^{\prime}} .
$$


Würde man x noch grösser wählen, so würde schon Öl mit der schwereren Flüssigkeit aus dem Rohr austreten. Macht man den Abstand $x$ dagegen zu klein, so hat die Emulsion häufig nicht Zeit, sich zu trennen und es fliesst Öl zugleich mit der schwereren Flüssigkeit über den Rand herab.

Der Abstand $x$ muss demnach für jedes Paar von Flüssigkeiten aus den specifischen Grewichten zuerst annähernd berechnet, dann aber noch empirisch so regulirt werden, dass die beste Wirkung erzielt wird.

Die Lage der Grenzschicht im Separator, entsprechend dem Abstande $y$, ist unabhängig von dem Mischungsverhältniss der beiden Flüssigkeiten.

Aber auch bei richtiger Einstellung des Apparates kommt es vor, dass die Emulsionen nicht genügend geschieden werden. Namentlich beim Waschen fetter Öle mit Laugen bleiben häufig grössere Fettmengen in den wässrigen Flüssigkeiten suspendirt. Ja es gibt Emulsionen, welche Ekenberg als "kritische" bezeichnet, welche durch den Separator überhaupt nicht geschieden, sondern im Gegentheile noch stabiler gemacht werden. In solchen Fällen erzielt man meist durch Abänderung des Mischungsverhältnisses der Concentration der Waschflüssigkeit oder durch Arbeiten in der Wärme zufriedenstellende Resultate. Andererseits bringt oft ein mässiger Zusatz von Kochsalz oder Glaubersalz Abhilfe, doch darf der Salzgehalt des Waschwassers nicht so gross sein, dass die Seifen ausgesalzen werden, welche ausgewaschen werden sollen.

Man kann daher eine für eine bestimmte Fettart aufgestellte Waschbatterie nicht ohne weiteres für ein anderes Fett benutzen. Auch bei der Anwendung warmer Waschflüssigkeiten wird man nicht über die gerade nothwendige Temperatur gehen, weil das Aroma mancher Öle durch zu starkes Erwärmen leidet.

Zur Wiedergewinnung der in den Waschwässern enthaltenen Fettsäuren sammt geringen Mengen emulgirten Öles säuert man an, worauf das Fett an die Oberfläche steigt. Auch die bei den Waschungen mit Wasser oder verdünnten Säuren in Emulsion verbliebenen geringen Ölmengen werden nach einer der von Ekenberg bereits beschriebenen $\mathrm{Me}$ thoden wiedergewonnen und in das Rohöl zurückgegossen oder als Secundafett an die Seifensieder abgegeben.

Hat man eine Waschbatterie nach Ekenberg einmal im richtigen Gang, so bietet sie ausser der Möglichkeit, continuirlich $\mathrm{zu}$ arbeiten, noch folgende Vortheile:

1. Das Rohöl kann, wie es von den Pressen kommt, in der Regel direct verarbeitet werden, doch kann man, wo man es vortheilhaft findet, zuerst mit Wasser emulgiren und separiren, wobei die Zellreste u. dgl, vom Wasser mitgerissen werden.

2. Beim Raffiniren mit concentrirter Schwefelsäure werden 40 bis 60 Proc. Fett weniger verseift als bei dem gewöhnlichen Verfahren, das Product ist dementsprechend weniger sauer.

3. Der Separator scheidet die Schwefelsäure bis auf ca. 0,2 Proc. ab, was sonst nur durch mehrtägiges Stehen erreicht werden kann.

4. Der Rest von Schwefelsäure ist durch einmaliges, böchstens zweimaliges Waschen vollständig zu entfernen.

5. Behandelt man nach dem Rafiniren mit Schwefelsāure direct mit Alkali, so ist der Verbrauch an demselben, falls keine freie Fettsäuren vorhanden sind (Mineralöle), ein minimaler, weil eben nur 0,2 Proc. Schwefelsäure zurückgeblieben sind.

6. Die Ausbeute an reinem Öl ist bei richtig geleiteten Operationen in der Regel grösser als nach dem alten Verfahren.

7. Freie Fettsäuren können bis auf 0,05 bis 0,10 Proc. ausgewaschen werden.

\section{Zur Säurebestimmung im Essig.

$$
\text { Von }
$$

\section{Dr. L. Vanino.}

Baumann veröffentlichte in dieser Zeitschrift 1892 eine Reihe von Bestimmungen organischer und anorganischer Säuren mittels Jodkalium, jodsaurem Kalium und Wasserstoffsuperoxyd, welche von $\mathrm{Kux}$ (Z. anal. 1893) auf Veranlassung Baumann's einer eingehenden Prüfung unterzogen wurden. Unter Anderem bearbeitete $\mathrm{Kux}$ auch eine Essigsäurebestimmung, welche nach folgender Reaction verläuft:

$$
\begin{gathered}
5 \mathrm{~K} \mathrm{~J}+\mathrm{K} \mathrm{J} \mathrm{O}+6 \mathrm{CH}_{3} \mathrm{COOH}= \\
6 \mathrm{~J}+6 \mathrm{CH}_{3} \mathrm{COOK}+3 \mathrm{H}_{2} \mathrm{O} \\
2 \mathrm{~J}+\mathrm{K}_{2} \mathrm{O}=\mathrm{JOK}+\mathrm{JK} \\
\mathrm{JOK}+\mathrm{JK}+\mathrm{H}_{2} \mathrm{O}_{2}=2 \mathrm{KJ}+\mathrm{H}_{2} \mathrm{O}+\mathrm{O}_{2} .
\end{gathered}
$$

Wie man aus der Zusammenstellung seiner Resultate ersieht, gestattet das Verfahren eine rasche und genaue Werthbestimmung der Essigsäure und was ein besonderer Vorzug bei dieser Methode ist, ist der eine Umstand, dass sje bei allen Sorten von Essig angewandt werden kann, während die übliche Titrationsmethode mit $\mathrm{KOH}$ und Phenolphthaleïn beistark gefärbten Essigsorten unbrauchbar ist. Kux benutzt zur Bestimmung das von P. Wagner abgeänderte Azotometer. Wenn auch zugegeben werden muss, dass dieses gasanalytische Instrument Sicherheit gewährt, so möchte ich doch dem Praktiker für obigen Zweck die Benutzung des Apparates empfehlen, welchen ich seiner- 